\title{
1 HIV-1 founder variant multiplicity is determined by the infection stage of the 2 source partner
}

4 Ch. Julián Villabona-Arenas ${ }^{1,2}$, Matthew Hall ${ }^{3}$, Katrina A. Lythgoe ${ }^{3}$, Stephen G. Gaffney ${ }^{4}$, Roland R. 5 Regoes $^{5}$, Stéphane Hué ${ }^{1,2}$, Katherine E. Atkins ${ }^{1,2,6 *}$

$6{ }^{1}$ Department of Infectious Disease Epidemiology, Faculty of Epidemiology and Population Health,

7 London School of Hygiene and Tropical Medicine, London, UK

$8{ }^{2}$ Centre for Mathematical Modelling of Infectious Diseases, London School of Hygiene and Tropical

9 Medicine, London, UK

$10{ }^{3}$ Big Data Institute, Nuffield Department of Medicine, University of Oxford, Oxford, UK

$11{ }^{4}$ Division of Biostatistics, Yale School of Public Health, New Haven, USA

$12{ }^{5}$ Institute of Integrative Biology, Department of Environmental Systems Science, ETH Zurich, Zurich,

13 Switzerland

$14{ }^{6}$ Centre for Global Health, Usher Institute of Population Health Sciences and Informatics, The University

15 of Edinburgh, Edinburgh, UK

$16 *$ corresponding author: Katherine.Atkins@ed.ac.uk

\section{Abstract}

18 During sexual transmission, the large genetic diversity of HIV-1 within an individual is frequently

19 reduced to one founder variant that initiates infection ${ }^{1}$. Understanding the drivers of this bottleneck is

20 crucial to develop effective infection control strategies ${ }^{2}$. Genetic characteristics of the potential founder

21 viruses and events in the recipient partner are both known to contribute to this bottleneck, but little is

22 understood about the importance of the source partner ${ }^{3}$. To test the hypothesis that the source partner

23 affects the multiplicity of HIV founder variants, we developed a phylodynamic model calibrated using

24 genetic and epidemiological data on all existing transmission pairs for whom the direction of transmission

25 and the infection stage of the source partner are known. Our results demonstrate the importance of

26 infection stage of the source partner, and not exposure route, in determining founder variant multiplicity. 
27 Specifically, acquiring infection from someone in the acute (early) stage of infection increases the risk of

28 multiple variant transmission when compared with someone in the chronic (later) stage of infection. This

29 study provides the first direct test of source partner characteristics to explain the low frequency of

30 multiple founder strain infections and can inform clinical intervention study design and interpretation.

\section{Main}

32 Sexual transmission of HIV-1 results in a viral diversity bottleneck due to physiological barriers as well

33 as viral or cellular constraints that prevent most genetic variants within the source partner from

34 establishing onward infection ${ }^{1,4,5}$. Indeed, this diversity bottleneck results in around three quarters of new

35 infections being founded by a single genetic variant ${ }^{2,6-10}$. The extent of genetic diversity transmitted to a

36 new partner is a crucial determinant in understanding the efficacy of putative vaccines and may shed light

37 on the transmission of drug resistance to treatment naive individuals.

The factors leading to the diversity bottleneck during sexual transmission can be broadly categorized as those determined by the source partner — such as viral load and viral diversity available for transmission,

41 those determined by the recipient partner — such as target cell type and availability in the genital or rectal

42 mucosa (e.g. ${ }^{3,5,11}$ ), and those connected with viral characteristics — such as glycosylation profiles and cell

43 tropism (reviewed in ${ }^{12}$ ). While the impact of the recipient partner and the characteristics of transmitted

44 variants have been widely discussed, little is known about how the source partner affects the viral

45 diversity bottleneck. In particular, despite the importance of infection stage as a driver of HIV

46 transmission - that is, the length of time between the source partner becoming infected and transmission

47 to their partner - there is no empirical evidence to suggest how this influences the viral diversity

48 bottleneck. This gap has arisen because analyses are routinely conducted on individuals without

49 information on the partner from whom they acquired infection. Phylogenetic analyses now offer a

50 possible solution to this impasse. 
52 Phylogenetic trees are representations of the ancestral relationships of organisms with the tips of the tree

53 representing those that are sampled, the internal nodes their inferred common ancestors, and the branches

54 as the evolutionary pathways between these actual and inferred individuals. When phylogenetic trees are

55 constructed using sequence data from both partners in an HIV transmission pair, the relationship between

56 the evolutionary histories of both set of viral samples may reflect epidemiological relationships between

57 the two individuals ${ }^{13-15}$. Previous modelling studies suggest that by assigning the relationship between

58 the evolutionary histories of both partners as one of three topology classes-monophyletic-monophyletic

59 (MM), paraphyletic-monophyletic (PM), or a combination of paraphyletic and polyphyletic (PP)—

60 epidemiological information can be inferred, such as the direction of transmission ${ }^{13}$, as well as

61 evolutionary information, such as determining the number of transmitted variants ${ }^{16}$. That is, the number

62 of monophyletic clusters in a PM (one) or PP (more than one) tree can be interpreted as the minimum

63 number of transmitted lineages (Fig. 1A). In practice, however, many factors may influence

64 epidemiological interpretations from phylogenetic trees such as sampling times, sampling density of the

65 viral populations and phylogenetic signal ${ }^{17,18}$.

67 Here we present a data-driven phylodynamic approach to overcome these empirical and methodological

68 issues to evaluate the impact of the source partner's infection stage and route of exposure on the HIV

69 diversity bottleneck (Fig. 1B,C). We first retrieved all available genetic and epidemiological information

70 from published HIV sexual transmission pairs where the direction of transmission is known, and kept for

71 further analysis those pairs for whom transmission could be classified as having occurred in the source

72 partner's acute stage ( $\leq 90$ days after his/her infection) or chronic stage (later than 90 days after his/her

73 infection). After further stratifying pairs into heterosexual (HET) and men-who-have-sex-with-men

74 (MSM) risk groups, we found a significant difference in the timing of transmission between the two risk

75 groups. Specifically, 10 of 36 MSM pairs were the result of acute stage transmission compared with 1 of

7676 of HET pairs (Fig. 2). 


\section{A}

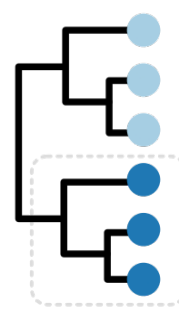

Monophyletic-Monophyletic (MM)
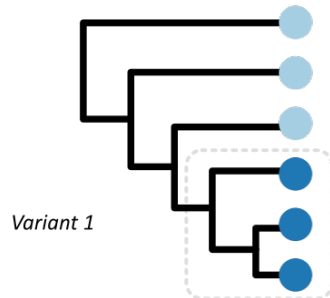

Paraphyletic-Monophyletic

(PM)

Recipient

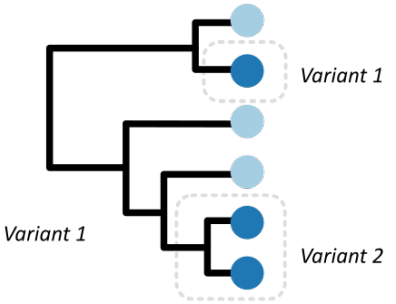

Paraphyletic-Polyphyletic

(PP)

\section{C}

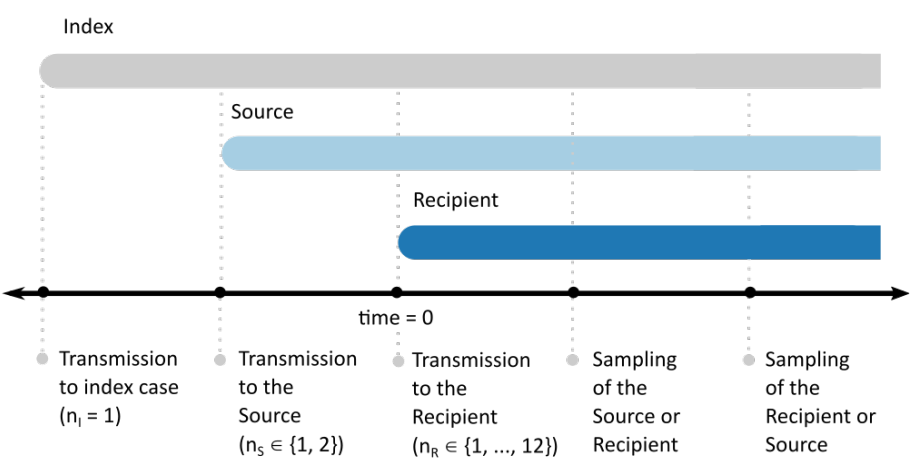

B

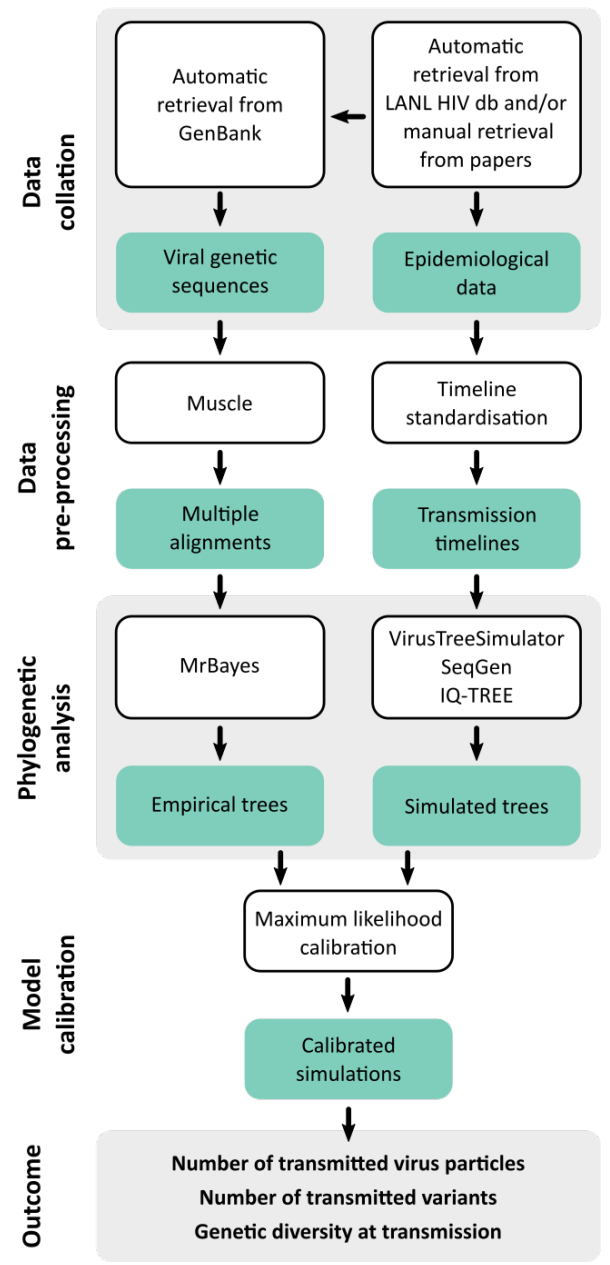

FIGURE 1: Methods schematics. A) Phylogenetic tree topology class of known transmission pairs that have previously been used as a proxy for calculating the minimum number of founder variants transmitted to the recipient: trees of class MM and PM both suggest a minimum of one founder variant while trees of class PP suggest a multiple founder variants, with the minimum number of founder variants being the number of recipient clades embedded in PP trees (here shown as two). B) Pipeline of phylodynamic analysis (LANLdb, Los Alamos National Laboratory HIV sequence database) where teal represents data or analysis output and white represents methods and analysis. An example of a standardised transmission timeline for a known source-recipient pair is provided in panel C. C) Schematic of the transmission pair model simulation that shows the transmission and sampling timelines. The simulated number of virus particles transmitted to the index case, and the source and recipient partners $\left(n_{\mathrm{I}}, n_{\mathrm{S}}, n_{\mathrm{R}}\right.$ respectively) are shown on the transmission events timeline. 
We then performed Bayesian phylogenetic tree reconstruction on the genetic sequences of the transmission pairs and classified the topology class of each tree in the posterior distribution as monophyletic-monophyletic (MM), paraphyletic-monophyletic (PM) or paraphyletic-polyphyletic (PP). The most likely topology class was PM (65\% and 61\% for HET and MSM, respectively), but with a

93 higher number of PP trees in the MSM group ( $P=0.056$, Fig. 2). This result has previously been reported

94 as indicative of a higher number of founder variants for MSM ${ }^{16}$. However, when we stratify the topology

95 class by whether the source partner was in acute or chronic infection at the time of transmission, our

96 results indicate that the infection stage of the source is the primary driver for any observed differences in

97 topology class. Specifically, there is no difference between the HET and MSM groups in the PM/PP

98 topology class ratio when transmission occurs in the chronic stage of infection $(P=0.570)$. Note that only

99 one HET transmission occurs during the acute stage, and the topology class for this pair is PP. These

100 results remain qualitatively consistent when only data were analysed from the $66 \%$ of transmission pairs

101 for whom the posterior trees gave a certainty of over 95\% for the most frequent topology class (Fig. S3).

102 These results indicate that infection stage of the source partner, and not risk group per se, influences the 103 diversity bottleneck at transmission.

105 To test whether these empirical findings are indicative of a smaller diversity bottleneck in the chronic 106 stage of HIV infection, we developed a phylodynamic framework in which we simulated the 107 epidemiologic characteristics of each HET and MSM transmission pair, the timing of their sequence 108 sampling, the transmission of virus particles, and the within-host genetic evolution in both the source and 109 recipient (Fig. 1B). Specifically, using the epidemiological information from the transmission pairs, we 110 simulated phylogenies under a coalescent model before generating genetic sequences from these 111 simulations and performing Maximum Likelihood (ML) phylogenetic reconstruction on these simulated 112 sequences. We classified each of these simulated trees as MM, PM or PP and determined the frequency of 113 each topology class for each simulated transmission pair across all the simulated sequences. However, as 114 we could not directly observe the number of virus particles that are transmitted between source and 


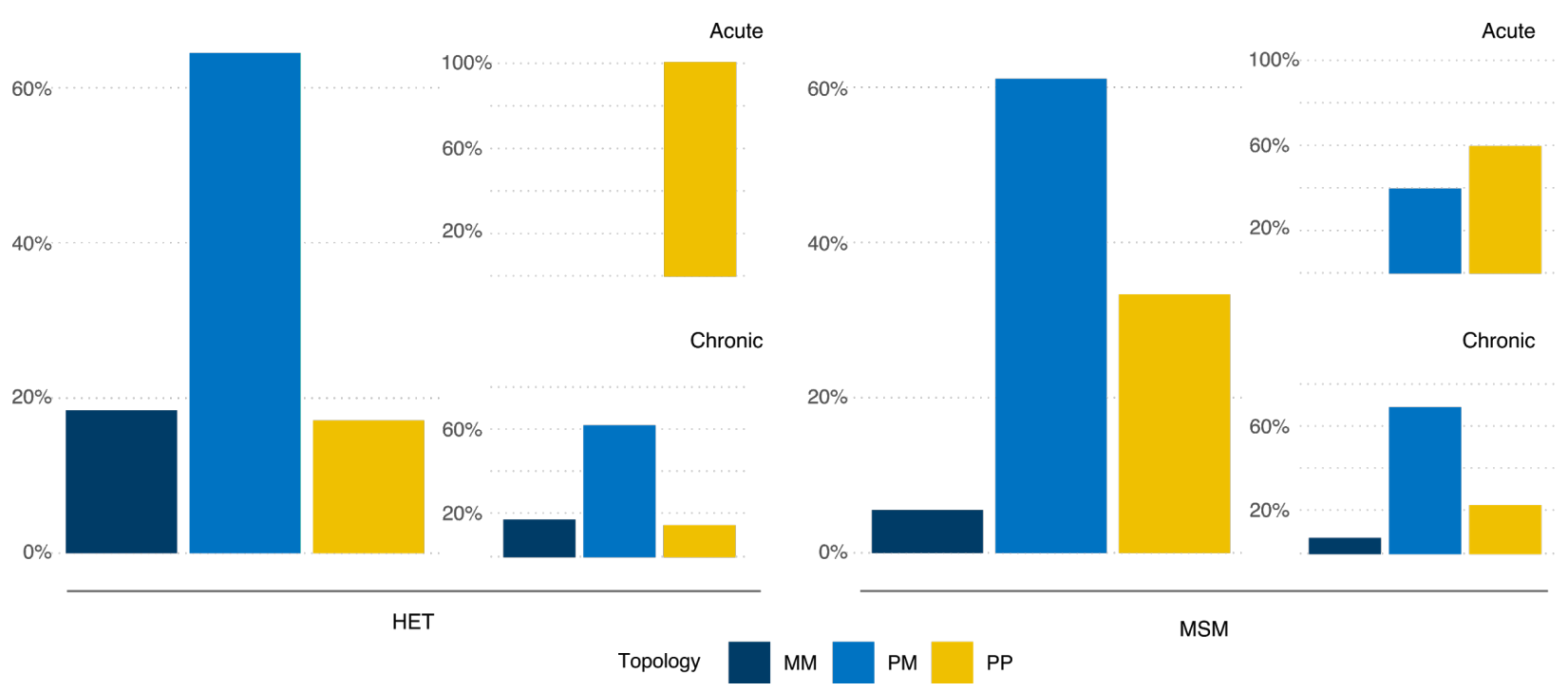

FIGURE 2: Phylogenetic findings from the empirical transmission pairs. Fraction of phylogenetic tree topology class (MM: Monophyletic-Monophyletic, PM: Paraphyletic-Monophyletic and PP: Paraphyletic-Polyphyletic) where each tree topology class is classified as the most frequent topology class of each posterior distribution per transmission pair. Results are stratified by risk group: 76 heterosexual (HET) pairs and 36 men-who-have-sex-withmen (MSM) pairs) and infection stage of the source partner at transmission (11 acute pairs defined as $<90 \mathrm{~d}$ post infection and 101 chronic pairs defined as $\geq 90 \mathrm{~d}$ post infection).

recipient, we repeated the simulation of phylogenetic trees for each transmission pair under a range of

124 plausible values of virus particles transmitted. By fitting the simulation output topology class distribution to the topology class distribution from the empirical phylogenetic trees using maximum likelihood inference, we then determined the most likely number of transmitted virus particles for each transmission pair and used this best fit model for further analysis. Note that two or more virus particles may have the same genetic sequence, and would constitute a single founder variant (or haplotype), discussed later. 
particle is transmitted ( $25 \%$ of pairs, Fig. 3A). While there is a high degree of confidence in the result when one particle is transmitted, there is often uncertainty around the exact number when multiple

134 particles are transmitted (Fig. 3A). Importantly, we found acute stage transmissions are less likely to lead 135 to single particle infections compared with chronic stage transmissions $(27 \%$ vs. $80 \%, P=0.0005)$. The 136 topology class of the simulated phylogenetic trees is strongly influenced by the number of virus particles

137 being transmitted (Fig. 3B). PM trees are more commonly found in the pairs that are better described by a 138 model with a single transmitted virus particle (81\%) whereas PP trees appeared more often when multiple 139 particles are likely to have been transmitted (86\%).

141 For each transmission pair, we then simulated the genetic sequences of the transmitted viral population 142 under the best fit virus particle model and calculated the most likely number of transmitted variants for 143 each transmission pair. The median number of variants transmitted across all pairs is 1 (range: 1-11, Fig.

144 4A). Using the full distribution of founder variant multiplicity for each pair, we also calculated the 145 probability that a single founder variant was transmitted to the respective recipient. Our results suggest 146 that across all pairs in both risk groups, the mean probability of observing one founder variant is 0.73 .

147 Stratifying by risk group, we find there is a higher probability that one variant founds HET infections than 148 MSM infections (a geometric mean of 0.80 vs. 0.63, Fig. 4B). However, these risk group differences 149 mostly disappear when we stratify the results by the infection stage of the source. Here, for example, 150 when only chronic stage transmissions are considered, the probability of one founding variant is a little 151 higher for MSM transmissions than for HET transmissions (means of 0.80 vs 0.71 ), and the pairwise 152 diversity at transmission is similar between both groups (Fig. 4C). In contrast, when stratifying solely by 153 infection stage of the source partner, we find that transmission during the acute stage has a much lower 154 probability of one founder variant than during the chronic stage (means of 0.40 vs. 0.77 ) with a higher 155 median number of variants transmitted, when only the most likely multiplicity for each pair is considered 156 (2 vs. 1, Fig. 4A). Nonetheless, if multiple variant transmission does occur, our results suggest that the 

measure during this later stage of infection (Fig. 4C).

A

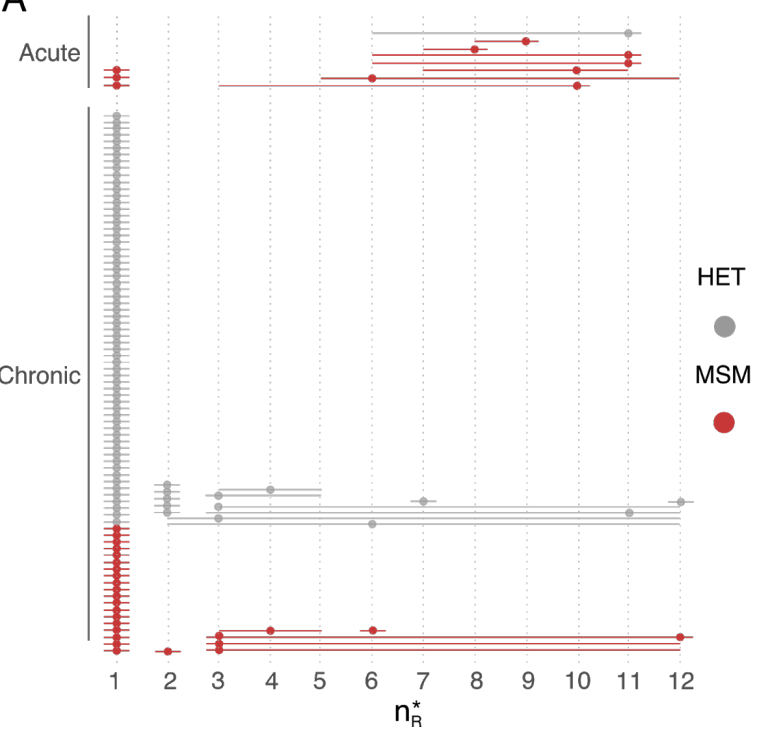

B

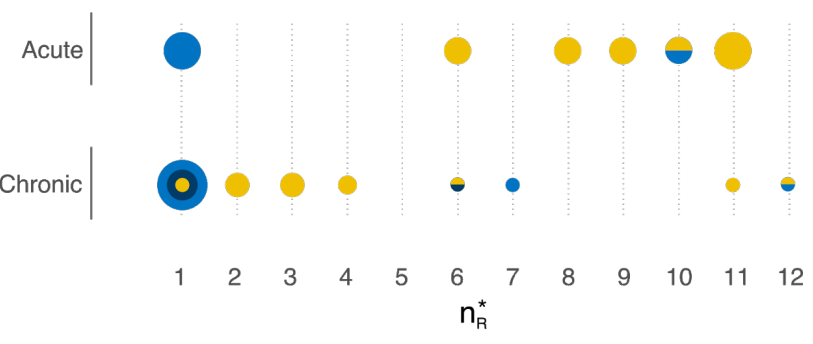

Frequency

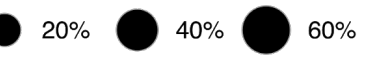

Topology $\square$ MM $\square$ PM $\square$ PP

FIGURE 3: The estimated number of transmitted virus particles for the 112 transmission pairs. The estimates

of transmitted virus particles for each transmission pair were calculated by choosing the model simulation that

generated a phylogenetic tree topology class distribution (that is, the number of MM, PM and PP trees constructed

from the simulated genetic sequences) that best matched the topology class distribution from the phylogenetic trees

constructed from the empirical genetic sequences. A) Maximum likelihood number of virus particles founding recipient infections, $n_{\mathrm{R}}^{*}$, for each pair (stacked points) with $95 \%$ confidence intervals (lines) grouped by stage of infection (acute, 11 pairs or chronic, 101 pairs) and risk group (76 heterosexual pairs, HET and 36 men-who-havesex-with-men pairs, MSM). B) Maximum likelihood number of virus particles founding recipient infections coloured by topology class of the phylogenetic tree constructed from the simulated genetic sequences.

171 From these results, therefore, there is approximately double the chance of multiple variant transmission

172 during acute stage infection across both risk groups (relative risk $=0.52$ ). Assuming that transmission

173 risk is weighted towards early transmission such that half of all index case to source partner transmissions 
174 occur after 90 days of index case infection leads to qualitatively similar results (Supplementary

175 Information). Similarly, calibrating the simulation model to bootstrapped samples rather than Bayesian

176 posterior distributions leads to similar results (Supplementary Information).

178 Our results suggest that there is an association between tree topology class and multiple variant

179 transmission, with 95\% of MM and PM trees being due to one founder variant (Fig. 4D). However, the

180 number of embedded recipient clades is not always a proxy for the minimum number of founder variants

181 transmitted. For example in chronic stage transmission, 11\% of PP topology class trees were due to single

182 variant transmission (Fig. 4D). Across both infection stages, we find that if MM, PM or PP is assigned as

183 the most likely tree topology class, then $92 \%, 96 \%$ and $15 \%$ of transmissions are due to a single founder

184 variant, respectively.

186 We have used a combination of empirical data and phylodynamic model simulation to evaluate the role of

187 infection stage at transmission and route of transmission on the number of virus particles transmitted

188 during sexual HIV exposure. This makes three important advances on previous work. First, it is the first

189 empirically-based study that fits a model to data to understand the role of the source partner in

190 establishing infection. Second, while we use previously developed topology classification of phylogenetic

191 trees to understand HIV transmission pairs, we extend this methodology by calibrating a phylodynamic

192 model to empirical data. This new approach provides a means to validate the untested assumption that the

193 number of embedded recipient partner lineages in a phylogenetic tree directly corresponds to the

194 minimum number of founder variants transmitted. Third, our phylodynamic model explicitly incorporates

195 virus particle number and the identity of genetic sequences. Previous work has shown that the multiplicity

196 of founder variants has little impact on the topology class of the phylogenetic tree when only overall

197 genetic diversity, rather than sequence identity, is tracked ${ }^{13}$. 
medRxiv preprint doi: https://doi.org/10.1101/19013524; this version posted December 4, 2019. The copyright holder for this preprint (which was not certified by peer review) is the author/funder, who has granted medRxiv a license to display the preprint in perpetuity. It is made available under a CC-BY 4.0 International license.

A
Acute
Chronic
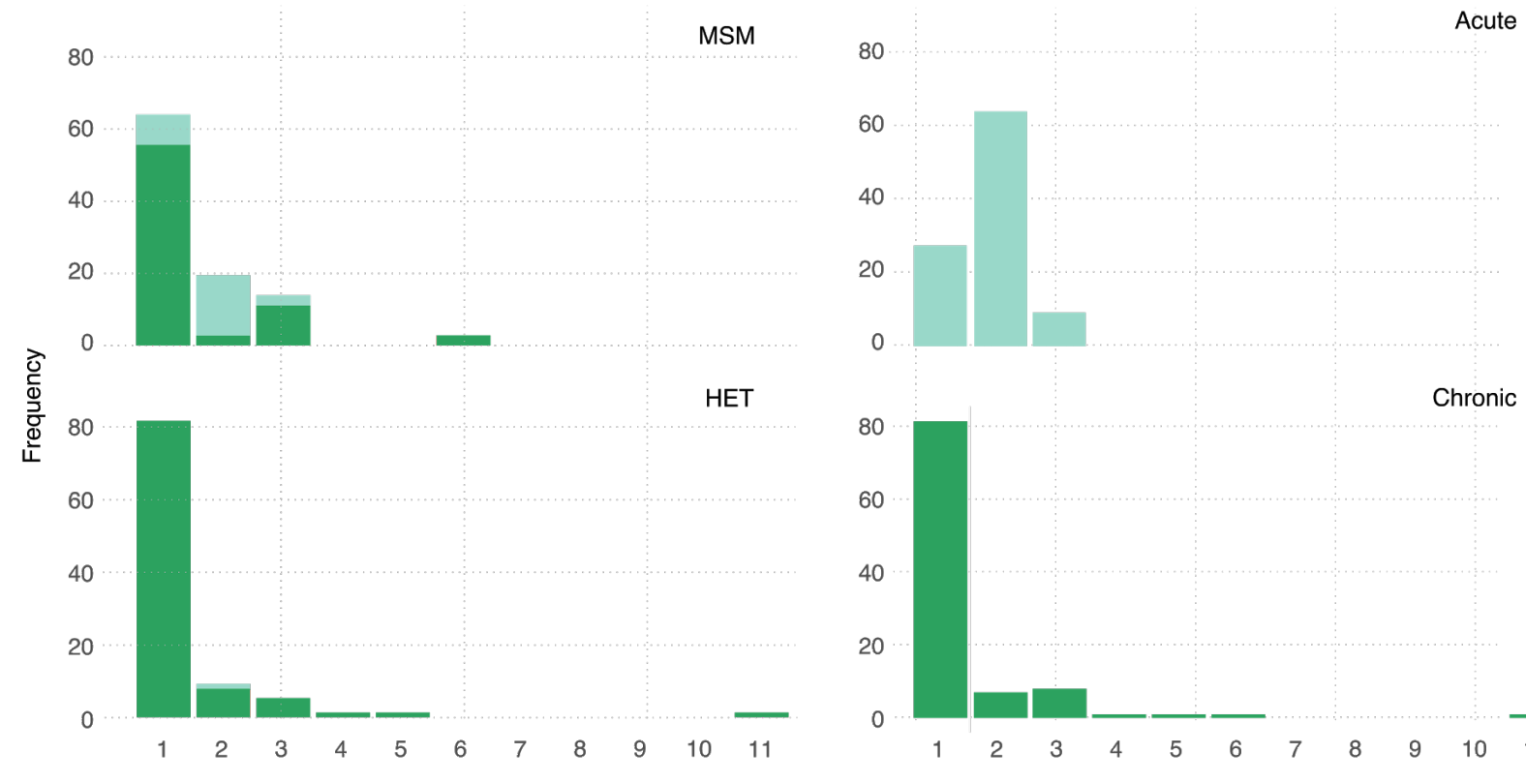

Founding variants

B

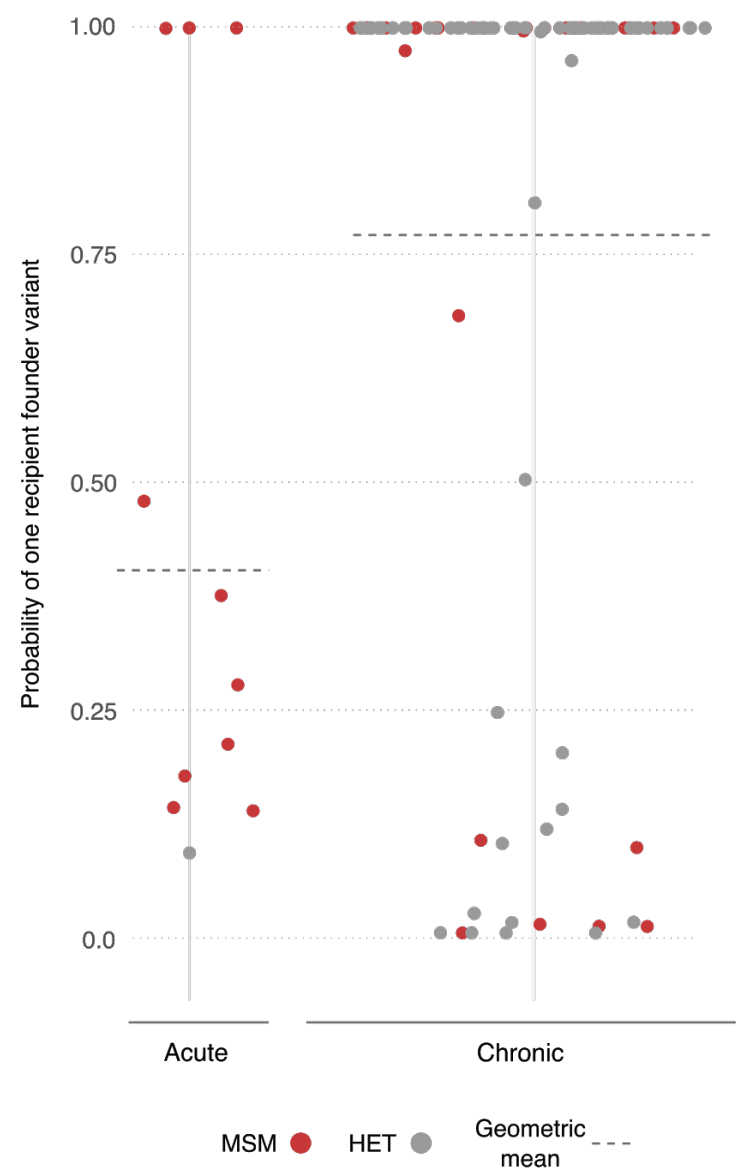

C

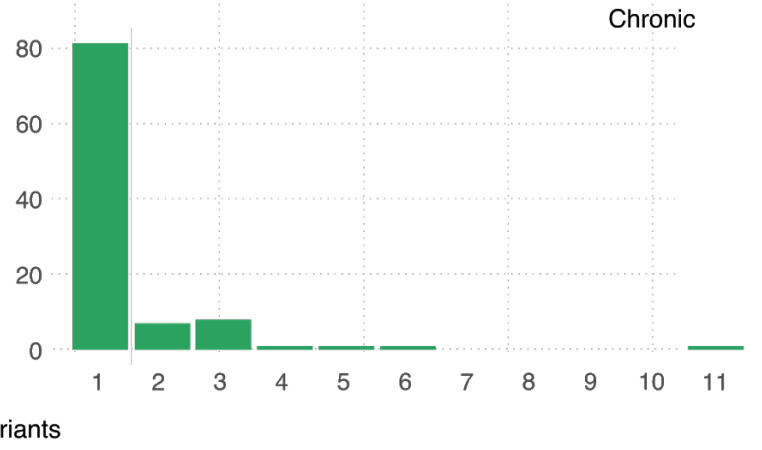

Acute

Chronic

20

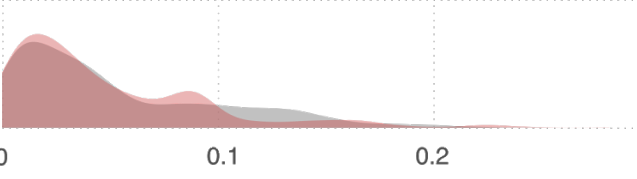

Maximum Nucleotide Diversity

D

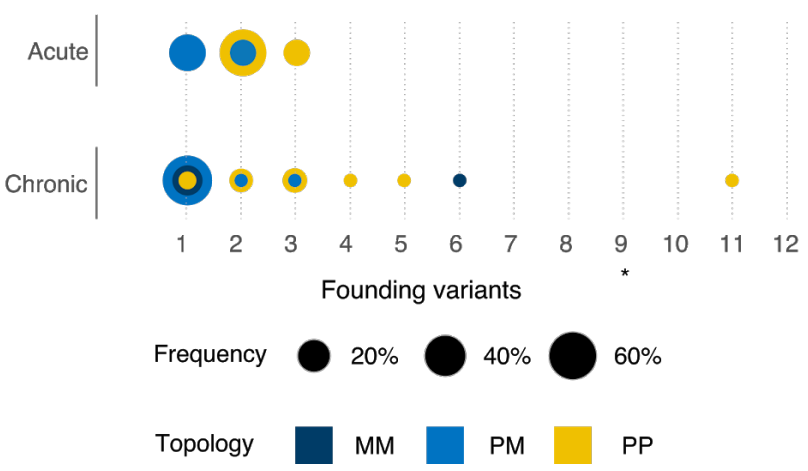


FIGURE 4: Phylogenetic findings from the calibrated simulations. A) Frequency of multiplicity of founding

variants for transmission pairs by infection stage of source partner at transmission and risk group. The multiplicity is stratified by infection stage of the source partner at transmission. C) Probability density distribution of maximum diversity (proportion of sites that differ) in the recipient partner across all simulations with more than one haplotype stratified by infection stage of the source at transmission. D) Number of founding variants coloured by topology class of the phylogenetic tree constructed from the best fit model of the simulated genetic sequences.

The relative importance of acute and chronic stages of HIV in determining both the number of virus particles and the number of variants transmitted is consistent with a recent modelling study ${ }^{19}$. However, our study finds higher proportions of infections initiated by multiple variants overall during these two

211 stages. This difference is likely due to the assumptions related to how the stages of infection are defined

212 as well as the relative importance of transmission during late infection. Specifically, the previous

213 modelling study finds that two thirds of multiple variant transmission occurs during the pre-AIDS stage of

214 infection which is assumed to have both a high viral load and large haplotype diversity. If later stages of

215 infection account for disproportionately less transmission then the previous model would predict higher

216 proportions of multiple variant transmission in both the acute and chronic stages of infection, becoming

217 more consistent with empirical estimates from our analysis. By contrast, our study is agnostic about the

218 relative importance of early and late transmission and does not differentiate between chronic and a pre-

219 AIDS stage of infection, which can not easily be identified through analysis of empirical data.

221 Data from four of the MSM transmission pairs in this study have previously been used to estimate the

222 number of variants founding infection using a combination approach of single genome amplification

223 (SGA), direct amplicon sequencing and mathematical modelling ${ }^{9}$ This previous analysis broadly agrees

224 with our results, with two recipients likely infected with one founder variant, one recipient with one to 
two founder variants and one recipient with two to three variants. Small differences likely arise because this study uses sequence data from both partners to evaluate the multiplicity of founder variants in the recipient partner. These extra data can be used to parameterize a mathematical model that accounts for the evolutionary relationship between the virus samples from both partners, rather than relying solely on accumulating diversity. Specifically, neglecting the extent of genetic similarity between the source and recipient virus samples might misattribute borderline cases of diversity accumulation.

232 Our study finds a median of one founder variant and a maximum of 11, with little difference between 233 HET and MSM risk groups. When only multiple variant transmissions are considered, our study finds a 234 median of 2-3 founder variants. These values are consistent with a previous pooled analysis using results 235 from four analyses that used the current gold-standard SGA combination approach as above ${ }^{9}$.

237 There are two primary limitations to acknowledge. First, our model assumes a single transmission event

238 between each source and recipient partner. Without detailed knowledge of the transmission pairs, we 239 cannot distinguish between multiple infections each with a single variant and a single infection with 240 multiple variants. Second, our phylodynamic framework does not account for the effect of selection and

241 recombination. Specifically, selection, such as that for viruses which use the CCR5 co-receptor ${ }^{20}$, is

242 thought to occur at the point of transmission, although the strength may be dependent on the route of

243 transmission ${ }^{21}$.

245 Our study finds that HIV-1 founder variant multiplicity is determined by infection stage of source partner, 246 with higher founder variant multiplicity of HIV-1 in acute compared with chronic infections. These

247 findings stress the importance of the stage of infection at transmission as an important mediating or 248 confounding factor when assessing the role of sexual risk group in HIV epidemiology. 


\section{Methods}

\section{Data collation on linked transmission pairs}

251 We automatically retrieved all HIV sequence data for men-who-have-sex-with-men (MSM) and

252 heterosexual (HET) HIV transmission pairs for whom the direction of transmission is reported from The

253 Los Alamos National Laboratory (LANL) HIV sequence database up to February 2019, such that each

254 transmission pair comprise a 'source' and a 'recipient' partner (Supplementary Information). For each

255 partner in the transmission pair we collected the following clinical and epidemiological data: (i) date of

256 infection or time of infection prior to sampling, (ii) date of seroconversion or date of seroconversion prior

257 to sampling, (iii) Fiebig stage at the time of sampling, (iv) date of sampling or time of sampling prior to

258 infection, (v) number of sequences, (vi) genomic region, (vii) HIV subtype, and (viii) reported risk group.

259 For each set of these transmission pair data we estimated, relative to the transmission time to the recipient

260 partner (time $=0)$ : $(i)$ the time of transmission to the source partner, (ii) the time of the sampling of the

261 source partner, and (iii) the time of sampling for the recipient partner (Fig. 1, Supplementary

262 Information). We excluded all transmission pairs from further analysis for whom these three times could

263 not be determined, for whom a risk group was not provided, for whom either partner has fewer than five

264 sequences for all sampling times, or for whom the pair did not have a LANL cluster ID. For our base case

265 analysis, we used the longest available genomic region with five or more sequences per partner. If more

266 than one sampling time is available for any of the individuals, we selected the sample closest in time to

267 the recipient transmission.

\section{Empirical transmission pair analysis}

270 Tree reconstruction: For each of the included transmission pairs, we generated posterior sets of

271 phylogenetic trees. For this, we first constructed alignments using Muscle v3.8.31 ${ }^{22,23}$ with subtype

272 specific reference sequences retrieved from the LANL HIV sequence database. Using these alignments, 
273 we built phylogenetic trees with MrBayes 3.2.7 $7^{24,25}$ under the assumption of a general time-reversible

274 (GTR) nucleotide substitution model with the addition of invariant sites (I) and a gamma distribution of

275 site rates. We constrained sequence data to be monophyletic with respect to the reference sequences to

276 root the tree but ingroup relationships were unconstrained to avoid any topology class bias. We ran two

277 Markov chains each with 30 million iterations, from which we sampled every 3,000th after discarding the

278 first 50\% as burn-in which provided an average standard deviation of split tree frequencies of below 0.01

279 or an effective sample size of greater than 300. This gave an empirical posterior distribution of $N=5,000$

280 sample trees. In a sensitivity analysis, we tested the alternative method of using maximum likelihood

281 phylogenetic tree reconstruction with bootstrapping.

Empirical topology class: We classified each of the resulting phylogenetic trees in the posterior

284 distribution as either monophyletic-monophyletic (MM), or paraphyletic-monophyletic (PM), or paraphyletic-polyphyletic (PP), to reflect the cladistic relationship between the lineages from both

287 probabilities, $D_{k}$, denoting the frequency of each topology class within the $k$ th pair's posterior distribution $\mathrm{D}_{\mathrm{k}}=\left\{d_{k}(t)\right\}_{t \in T}=\{\operatorname{Pr}(t \mid k)\}_{t \in T}$ where $\sum_{t \in T} \operatorname{Pr}(t \mid k)=1$ and $T \in\{\mathrm{MM}, \mathrm{PM}, \mathrm{PP}\}$.

\section{Simulated transmission pair analysis}

291 We simulated the transmission of virus particles and within-host evolution, accounting for the 292 epidemiological characteristics for each transmission pair. For each transmission pair, we simulated a 293 chain of three HIV infections: (i) an unsampled index case who infected the source after three years of

294 their own infection during their chronic stage to reflect that the majority of both HET and MSM

295 transmission pairs transmitted during the chronic stage (101/112 pairs). In a sensitivity analysis we

296 accounted for the assumption that transmission rate may be higher during the acute stage, with half of the

297 index to source transmissions occurring after 90 days and the remaining half after three years, (ii) the 
source individual of the transmission pair, and (iii) finally the recipient individual of the transmission consistent with that parameterized by the PANGEA-HIV study with the following logistic model parameters: initial effective population size $\left(N_{0}\right)$ is 1 , viral generation time $(\tau)$ is 1.8 days, effective

304 population per year growth rate $(r)$ is 2.85022 , and time to half the carrying capacity of the viral the same number of tips as the number of retrieved sequences per partner and that is sampled at the respective sampling times for the source and recipient partner (Supplementary Information). For each recipient partner infection, we assume that a total of $n_{\mathrm{R}}$ virus particles founded the infection. For each simulation, we further assume a total of $n_{\mathrm{S}}$ virus particles founding infection of the source. We assume $n_{\mathrm{R}}$ takes values between one and a maximum of 12 and varied $n_{\mathrm{S}}$ between one and two (Supplementary

311 Information). We assume that the virus samples from each recipient is representative of the within-host

312 diversity, and that each founding virus particle has an extant lineage. Therefore, we first assigned each 313 sample (tip) of a phylogeny as a descendant of one of the $n_{\mathrm{R}}$ virus particles. If there were more than 12

314 samples then the remaining tips were assigned randomly to the $n_{\mathrm{R}}=12$ virus particles. If there were

315 fewer than 12 samples, then we constrained the number of founding virus particles, $n_{\mathrm{R}}$, to equal the 316 number of samples. For every transmission pair, and for each value of $n_{\mathrm{R}}$ and $n_{\mathrm{S}}$, we simulated 100 viral 317 phylogenies.

319 For every simulated viral phylogeny, we simulated transmitted sequences by adding dummy nodes with a 320 negligibly short branch length after the transmission time. We then simulated the evolution of nucleotide 321 sequences along the tree using Seq-Gen ${ }^{27}$ and a GTR + I + gamma substitution model. The length of the 322 simulated sequences and the evolutionary tree scaling rate match each transmission pair's empirical 
sequence data. For this, we used previously estimated empirically-derived within-host evolutionary rates root. Every transmission pair simulation produces a tip sequence alignment and a number of founder sequences equal to the number of transmitted particles.

Simulated topology class: We reconstructed a phylogeny using maximum likelihood inference in IQphylogeny was classified as either MM, PP or PP (Supplementary Information). Consequently, for each transmission pair $k$ and each transmissibility model (i.e. number of viral particles founding infection of the recipient $\left.n_{\mathrm{R}}\right)$, we generated a triplet of probabilities $\left.M_{k, n_{\mathrm{R}}}=\left\{m_{k, n_{\mathrm{R}}}\right\}_{t \in T}=\operatorname{Pr}\left(t \mid k, n_{\mathrm{R}}\right)\right\}_{t \in T}$ where $\sum_{t \in T} \operatorname{Pr}\left(t \mid k, n_{\mathrm{R}}\right)=1$ and $T \in\{\mathrm{MM}, \mathrm{PM}, \mathrm{PP}\}$.

\section{Transmissibility model calibration}

For each transmission pair, we chose the most likely value of $n_{\mathrm{R}}$ (the number of virus particles founding each recipient infection) by matching the posterior topology class from the empirical phylogenetic transmission trees with the simulated distribution of topology class. Specifically, for each transmission pair, $k$, we estimated the most likely number of viral particles founding each recipient infection $n_{\mathrm{R}}^{*}$ as the $n_{\mathrm{R}}$ that maximises the multinomial likelihood function $L_{k, n_{\mathrm{R}}}=\operatorname{Pr}\left(D_{k} \mid M_{k, n_{\mathrm{R}}}\right)=\frac{N !}{\prod_{t \in T}\left(N d_{k}(t)\right) !} \prod_{t \in T} m_{k, n_{\mathrm{R}}}(t)^{N d_{k}(t)}$. For each transmission pair $k$, we calculated

342 lower and upper confidence limits for $n_{\mathrm{R}}^{*}$ as the minimum and maximum values of $n_{\mathrm{R}}$ that satisfy $L_{k, n_{\mathrm{R}}}>L_{k, n_{\mathrm{R}}^{*}}-1.92$ and $L_{k, n_{\mathrm{R}}}<L_{k, \mathrm{n}_{\mathrm{R}}^{*}}+1.92$, respectively ${ }^{31}$. For each transmission pair $k$, we retain

344 the best fit model for further analysis such that there are $n_{\mathrm{R}}^{*}$ viral particles founding infection of the 345 recipient.

\section{Haplotype analysis}



model, we defined the random variables $F_{\mathrm{S}}^{k}$ and $F_{\mathrm{R}}^{k}$ as the number of haplotypes that found infection of the source and the recipient partners, respectively. We then calculated the probability of there being a single founder haplotype in the recipient, stratified by topology class of the simulated phylogenetic tree $(\mathrm{MM}, \mathrm{PM}, \mathrm{PP})$ and the number of founder haplotypes, $i$, in the source partner, $p_{i}^{k}(t)$, that is, $p_{i}^{k}(t)=$ $\operatorname{Pr}\left(F_{\mathrm{R}}^{k}=1 \mid F_{\mathrm{S}}^{k}=i, t\right)$. Next, we defined the probability of a single founder haplotype in the recipient as a function of a tree topology, $t, p^{k}(t)=\operatorname{Pr}\left(F_{\mathrm{R}}^{k}=1 \mid t\right)=p_{1}^{k} \operatorname{Pr}\left(F_{\mathrm{S}}=1\right)+p_{2}^{k} \operatorname{Pr}\left(F_{S}>1\right)$. By assuming that the source partners are randomly selected from the general MSM or HET population in which the probability of a single founder strain has been calculated to be approximately $0.7^{19}$, we set, $\operatorname{Pr}\left(F_{\mathrm{S}}=1\right)=$

Founder haplotype multiplicity by source partner infection stage: We stratified all the transmission pairs into two sets by the infection stage of the source partner. We classified the acute transmission set as those pairs for whom recipient infection is within 90 days of source infection (a set of $n_{\text {acute }}$ pairs), and the haplotype being transmitted to the recipient in each set as:

$$
\begin{gathered}
q_{\text {acute }}^{k}=\sqrt[n_{\text {acute }}]{\prod_{k \in \text { acute }} q^{k}} \\
q_{\text {chronic }}^{k}=\sqrt[n_{\text {chronic }}]{\prod_{k \in \text { chronic }} q^{k}}
\end{gathered}
$$




\section{Data availability}

370 All code and data are available at github.com/AtkinsGroup under the following repositories: data on the

371 transmission pairs and sequence alignments are provided at (TransmissionPairs_Data), code for retrieval

372 of transmission pair epidemiological data and metadata from Los Alamos National Laboratory HIV

373 sequence database (TransmissionPairs_LANLRetrieval), code for sequence retrieval from GenBank

374 (TransmissionPairs_GenBankRetrieval), code for phylodynamic analysis

375 (TransmissionPairs_PhylodynamicAnalysis), and code for topological classification

376 (TransmissionPairs_TreeTopologyAnalysis).

\section{Author contributions}

378 KEA conceived the study. CJVA, MH, KL, SH, KEA designed the study. CJVA performed the 379 experiments and analysed the data. CJVA, MH, KL, SH, KEA interpreted the data. SGG created new 380 software used in the study. KEA and CJVA drafted the manuscript, with critical revisions from MH, 381 RRR, KL, SH. All authors approved the final version of the manuscript.

\section{Acknowledgements}

383 CJVA and KEA were funded by an ERC Starting Grant (award number 757688) awarded to KEA. KAL

384 was supported by The Wellcome Trust and The Royal Society grant no. 107652/Z/15/Z. MH was funded 385 by The HIV Prevention Trials Network (grant number H5R00701.CR00.01) and The Bill and Melinda 386 Gates Foundation (grant number OPP1175094).

\section{References}


Retrovirology 14, (2017).

2. Tully, D. C. et al. Differences in the Selection Bottleneck between Modes of Sexual Transmission Influence the Genetic Composition of the HIV-1 Founder Virus. PLoS Pathog. 12, e1005619 (2016).

3. Keele, B. F. \& Estes, J. D. Barriers to mucosal transmission of immunodeficiency viruses. Blood 118, 839-846 (2011).

4. Geoghegan, J. L., Senior, A. M. \& Holmes, E. C. Pathogen population bottlenecks and adaptive landscapes: overcoming the barriers to disease emergence. Proc. Biol. Sci. 283, (2016).

5. Talbert-Slagle, K. et al. Cellular superspreaders: an epidemiological perspective on HIV infection

6. Keele, B. F. et al. Identification and characterization of transmitted and early founder virus envelopes in primary HIV-1 infection. Proc. Natl. Acad. Sci. U. S. A. 105, 7552-7557 (2008).

7. Salazar-Gonzalez, J. F. et al. Deciphering human immunodeficiency virus type 1 transmission and early envelope diversification by single-genome amplification and sequencing. J. Virol. 82, 39523970 (2008).

8. Abrahams, M.-R. et al. Quantitating the multiplicity of infection with human immunodeficiency

9. Li, H. et al. High Multiplicity Infection by HIV-1 in Men Who Have Sex with Men. PLoS Pathog. 6, virus type 1 subtype $\mathrm{C}$ reveals a non-poisson distribution of transmitted variants. J. Virol. 83, 3556e1000890 (2010).

10. Gnanakaran, S. et al. Recurrent Signature Patterns in HIV-1 B Clade Envelope Glycoproteins Associated with either Early or Chronic Infections. PLoS Pathogens 7, e1002209 (2011).

412 (2012).

12. Sagar, M. Origin of the transmitted virus in HIV infection: infected cells versus cell-free virus. $J$.

$$
\text { Infect. Dis. } 210 \text { Suppl 3, S667-73 (2014). }
$$

13. Romero-Severson, E. O., Bulla, I. \& Leitner, T. Phylogenetically resolving epidemiologic linkage. 
Proc. Natl. Acad. Sci. U. S. A. 113, 2690-2695 (2016).

14. Ratmann, O. et al. Inferring HIV-1 transmission networks and sources of epidemic spread in Africa with deep-sequence phylogenetic analysis. Nat. Commun. 10, 1411 (2019).

15. Wymant, C. et al. PHYLOSCANNER: Inferring Transmission from Within- and Between-Host Pathogen Genetic Diversity. Mol. Biol. Evol. 35, 719-733 (2018).

16. Leitner, T. \& Romero-Severson, E. Phylogenetic patterns recover known HIV epidemiological relationships and reveal common transmission of multiple variants. Nat Microbiol 3, 983-988

17. Rose, R. et al. Phylogenetic Methods Inconsistently Predict the Direction of HIV Transmission Among Heterosexual Pairs in the HPTN 052 Cohort. J. Infect. Dis. 220, 1406-1413 (2019).

18. Abecasis, A. B., Pingarilho, M. \& Vandamme, A.-M. Phylogenetic analysis as a forensic tool in HIV transmission investigations. AIDS 1 (2017).

19. Thompson, R. N. et al. Link between the numbers of particles and variants founding new HIV-1 infections depends on the timing of transmission. Virus Evol 5, vey038 (2019).

20. Beretta, M. et al. Phenotypic properties of envelope glycoproteins of transmitted HIV-1 variants from patients belonging to transmission chains. AIDS 32, 1917-1926 (2018).

21. Carlson, J. M. et al. HIV transmission. Selection bias at the heterosexual HIV-1 transmission bottleneck. Science 345, 1254031 (2014). complexity. BMC Bioinformatics 5, 113 (2004).

23. Edgar, R. C. MUSCLE: multiple sequence alignment with high accuracy and high throughput. Nucleic Acids Res. 32, 1792-1797 (2004).

437 24. Ronquist, F. \& Huelsenbeck, J. P. MrBayes 3: Bayesian phylogenetic inference under mixed models. 438 Bioinformatics 19, 1572-1574 (2003).

439 25. Huelsenbeck, J. P. \& Ronquist, F. MRBAYES: Bayesian inference of phylogenetic trees.

$440 \quad$ Bioinformatics 17, 754-755 (2001). 
441 26. Ratmann, O. et al. Phylogenetic Tools for Generalized HIV-1 Epidemics: Findings from the 442 PANGEA-HIV Methods Comparison. Mol. Biol. Evol. 34, 185-203 (2017).

443 27. Rambaut, A. \& Grassly, N. C. Seq-Gen: an application for the Monte Carlo simulation of DNA

444 sequence evolution along phylogenetic trees. Comput. Appl. Biosci. 13, 235-238 (1997).

445 28. Alizon, S. \& Fraser, C. Within-host and between-host evolutionary rates across the HIV-1 genome.

446 Retrovirology 10, 49 (2013).

447 29. Nguyen, L.-T., Schmidt, H. A., von Haeseler, A. \& Minh, B. Q. IQ-TREE: a fast and effective 448 stochastic algorithm for estimating maximum-likelihood phylogenies. Mol. Biol. Evol. 32, 268-274 $449 \quad(2015)$.

450 30. Kalyaanamoorthy, S., Minh, B. Q., Wong, T. K. F., von Haeseler, A. \& Jermiin, L. S. ModelFinder: 451 fast model selection for accurate phylogenetic estimates. Nat. Methods 14, 587-589 (2017).

452 31. Cole, S. R., Chu, H. \& Greenland, S. Maximum likelihood, profile likelihood, and penalized 453 likelihood: a primer. Am. J. Epidemiol. 179, 252-260 (2014). 


\section{Supplementary Information for:}

\section{HIV-1 founder variant multiplicity is determined by infection stage of source 3 partner \\ 4 \\ 5 Ch. Julián Villabona-Arenas, Matthew Hall, Katrina A. Lythgoe, Stephen G. Gaffney, Roland R. Regoes, 6 Stéphane Hué, Katherine E. Atkins}

\section{Methods}

\section{Epidemiological data and sequence retrieval}

9 For the ease of replicating our results and using existing transmission pair data for other purposes, we

10 developed a Python script to automatically retrieve epidemiological and metadata for each transmission

11 pair from the Los Alamos National Laboratory HIV sequence database (LANLdb). This script downloads

12 the following data from both the source and recipient partners to a .csv file using as input the cluster and

13 patients ids from LANLdb: (i) time of infection, (ii) time of seroconversion, (iii) Fiebig stage at the time

14 of sampling, (iv) number of sequences, (v) genomic region, (vi) HIV subtype, (vii) reported risk group

15 and (viii) GenBank accession IDs.

16

17 Next we used the downloaded GenBank accession IDs to automatically retrieve (ix) viral genetic

18 sequences and (x) sampling dates (calendar dates) from GenBank using an R script. If information from

19 (i) to (x) were missing for any individual, we manually retrieved these values from the original

20 manuscripts where possible.

22 Completed datatables from these automatic and manual processes are provided at

23 github.com/AtkinsGroup. 


\section{Epidemiological data pre-processing}

26 For each transmission pair, we define time $=0$ as the time of recipient infection. We then calculated,

27 using the data table retrieved, i) the time of infection of the source, ii) the time of sampling of the source,

28 iii) the time of sampling of the recipient.

30 To estimate these times, we first calculated days from infection for both the source and the recipient

31 partners. When these values are not given explicitly, we calculated them from time since seroconversion

32 estimates or from Fiebig staging results. Specifically, we interpret (Cohen et al. 2010) seroconversion as

33 the individual reaching Fiebig stage III (ELISA positive) that occurs between 22-37 days after infection

34 (Cohen et al. 2010) and Fiebig stages I (viral RNA positive) and II (18-34) occurring 13 days and 28 days

35 after infection, respectively. For all the pairs where a range of possible values is calculated, and for when

36 a calendar month is provided, we incorporated the uncertainty around the infection and sampling times by

37 assuming all values in these ranges are equally plausible and uniformly sampled within these range to

38 account for the uncertainty.

40 For some pairs, the source was classified as 'recent' or 'late' at the time of transmission to the recipient

41 partner. In these cases, we do not have an exact point to call time $=0$. Therefore, for these pairs, for each

42 simulation, we sample with replacement the time between source and recipient infections from the other

43 pairs for whom we have previously classified as acute ( $<90$ days delay), and chronic ( 90 days or more

44 delay), sampling from the same risk group (MSM or HET) in each case.

46 All calculations, corresponding notes and final transmission times for each pair are provided at

47 github.com/AtkinsGroup and visualised in Fig. S1. 
medRxiv preprint doi: https://doi.org/10.1101/19013524; this version posted December 4, 2019. The copyright holder for this preprint (which was not certified by peer review) is the author/funder, who has granted medRxiv a license to display the preprint in perpetuity. It is made available under a CC-BY 4.0 International license.

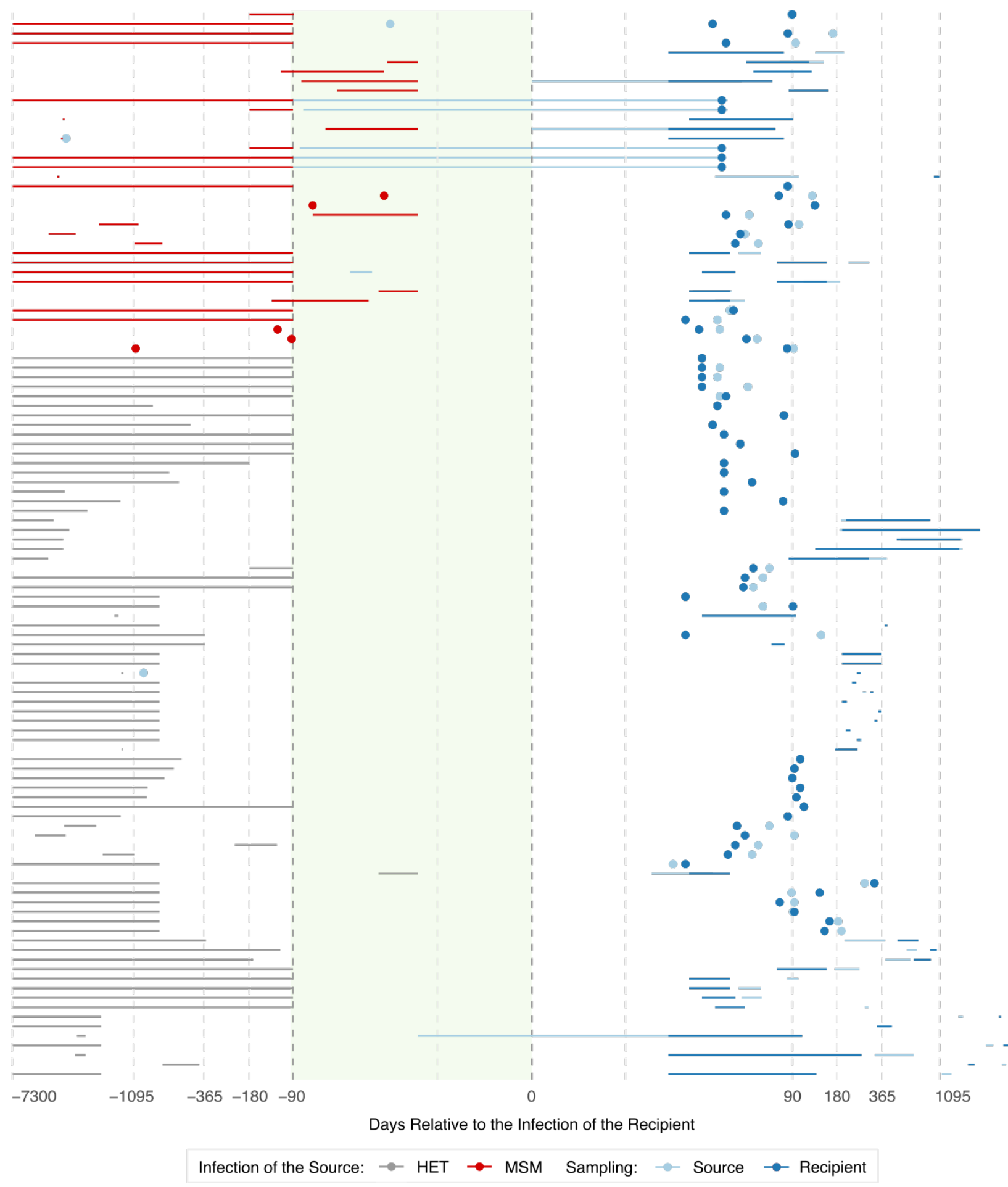

50 FIGURE S1: Infection and sampling times of the source and recipient for all the 112 transmission pairs

51 analysed. Individual points denote exact times and lines denote uniform uncertainty in times. Source partners 
52 points/lines overlapping the green shaded area correspond to transmission pairs for whom transmission occurs

53 during the acute stage.

\section{Results}

\section{Transmission pairs sequence data}

57 Our alignments are provided at github.com/AtkinsGroup.

58 On average, 22 (IQR 13-33) HIV sequences are obtained from the source and 21 (IQR 10-20) sequences

59 from the recipient for the MSM pairs, and 21 (IQR 12-25) and 18 (IQR 9-22) for the HET source and

60 recipient, respectively. All MSM sequence data belong to subtype B, while most heterosexual sequence

61 data belong to subtype C (49\%), followed by subtype B (22\%), subtype D (21\%), subtype A/A-like (7\%)

62 and unclassified subtype (1\%). A total of 7 (19\%) of the MSM pairs have near full genomes sequenced

63 and the remaining pairs had env available (mean $1653 \mathrm{nt}$, range 182-3827 nt). Ten (13\%) of the HET pairs

64 had near full genomes available, while 56 (75\%) pairs had env (mean $1321 \mathrm{nt}$, range 323-2582 nt), nine

65 (12\%) pairs had either pol or gag (mean $1484 \mathrm{nt}$, range 1375-1499 nt) and one pair had vif-LTR3 (4666

$66 \mathrm{nt})$ sequenced.

\section{Effect of number of founding virus particles in the source}

68 To assess whether the number of founding virus particles in the source partner affects the diversity of

69 sequences founding infection in the recipient, we model a scenario where the index case transmitted one,

70 two or six virus particles to the source partner at either one or three year(s) after infection. The source in

71 turn transmits 1 to 6 virus particles to the recipient at 30 days (acute) or 1095 days (chronic) later. The

72 simulation produces a dated viral phylogeny with tips sampled at either 30 (early) or 1065 days (late).

73 We model $1 \mathrm{~kb}$ nucleotide sequences along the simulated viral phylogenies using the same method as in 
74 the main text.

76 The genetic variation rapidly and steadily increases over time-the maximum diversity among

77 transmitted haplotypes to the recipient was higher when the index case was infected for longer and the

78 transmission to the recipient occurs during the chronic stage of the source (Fig. S2). When the source has

79 more than one founding particle, this leads to a bimodal distribution of maximum diversity among

80 transmitted variants within the recipient. The first and second mode represent maximum diversity when

81 drawing the recipient founder haplotypes from either one or more than one viral population within the

82 source, respectively. However, increasing the number of founding virus particles to more than two within

83 the source only increases the density around the second mode without affecting the range of the maximum

84 diversity distribution. This consistency occurs because increasing the number of founding virus particles

85 in both transmission partners, increased the probability of drawing founding variants from different

86 genetic pools in the source. However the average maximum diversity of the founder variants does not

87 change because the source genetic pools evolved at the same rate and under the same evolutionary

88 constraints with no selective advantage. This leads to genetic pools with equivalent cumulative genetic

89 change but distinct identity. Taking this into account, we chose to model one or two founding virus

90 particles within the source partner as we were interested in capturing some degree of variation in the

91 transmitted haplotypes rather than multiple genetic identities per se. 
medRxiv preprint doi: https://doi.org/10.1101/19013524; this version posted December 4, 2019. The copyright holder for this preprint (which was not certified by peer review) is the author/funder, who has granted medRxiv a license to display the preprint in perpetuity.

It is made available under a CC-BY 4.0 International license.

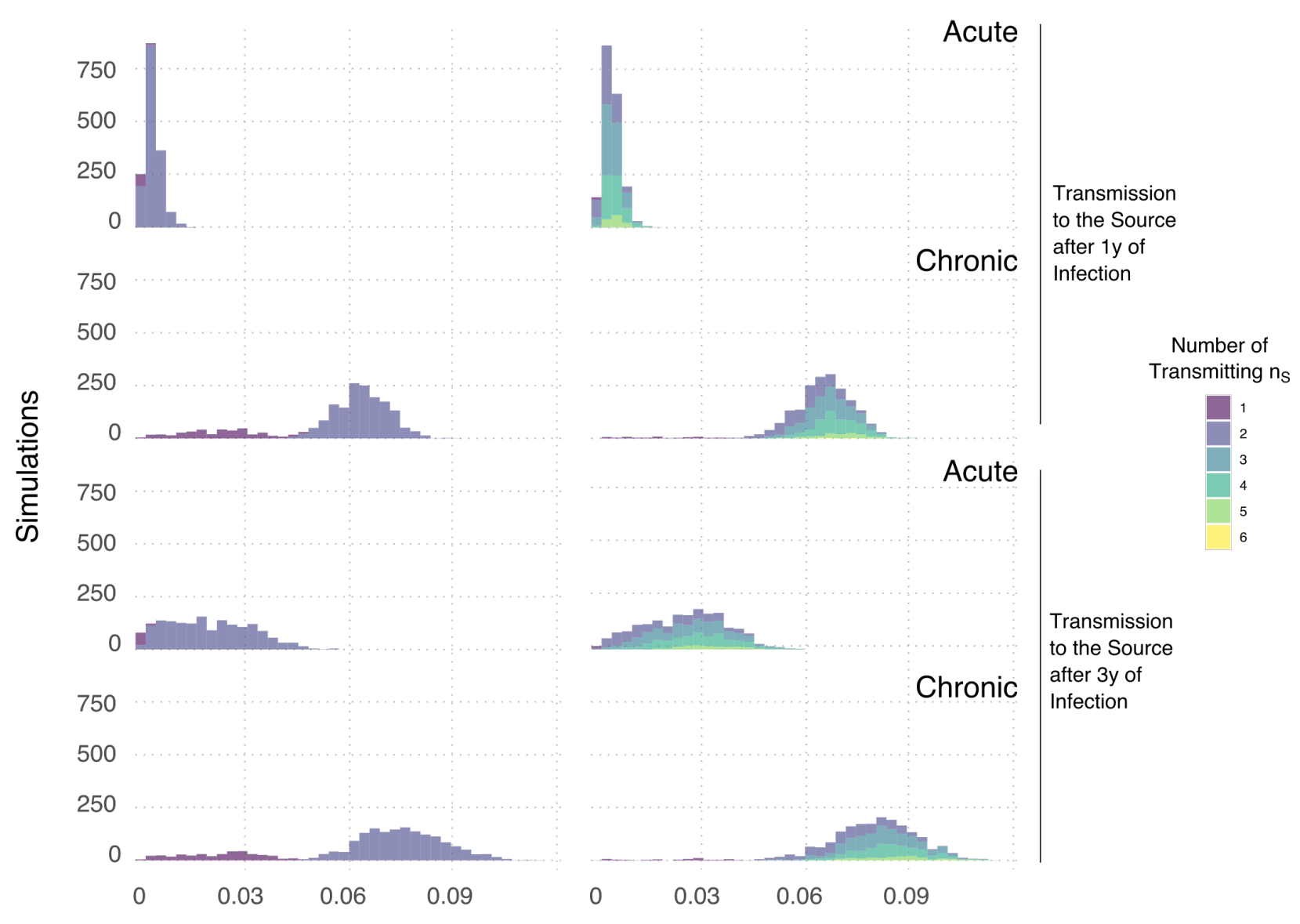

93 FIGURE S2: Effect of number of founding virus particles in the source.

94

95

96

97 
medRxiv preprint doi: https://doi.org/10.1101/19013524; this version posted December 4, 2019. The copyright holder for this preprint (which was not certified by peer review) is the author/funder, who has granted medRxiv a license to display the preprint in perpetuity.

It is made available under a CC-BY 4.0 International license.
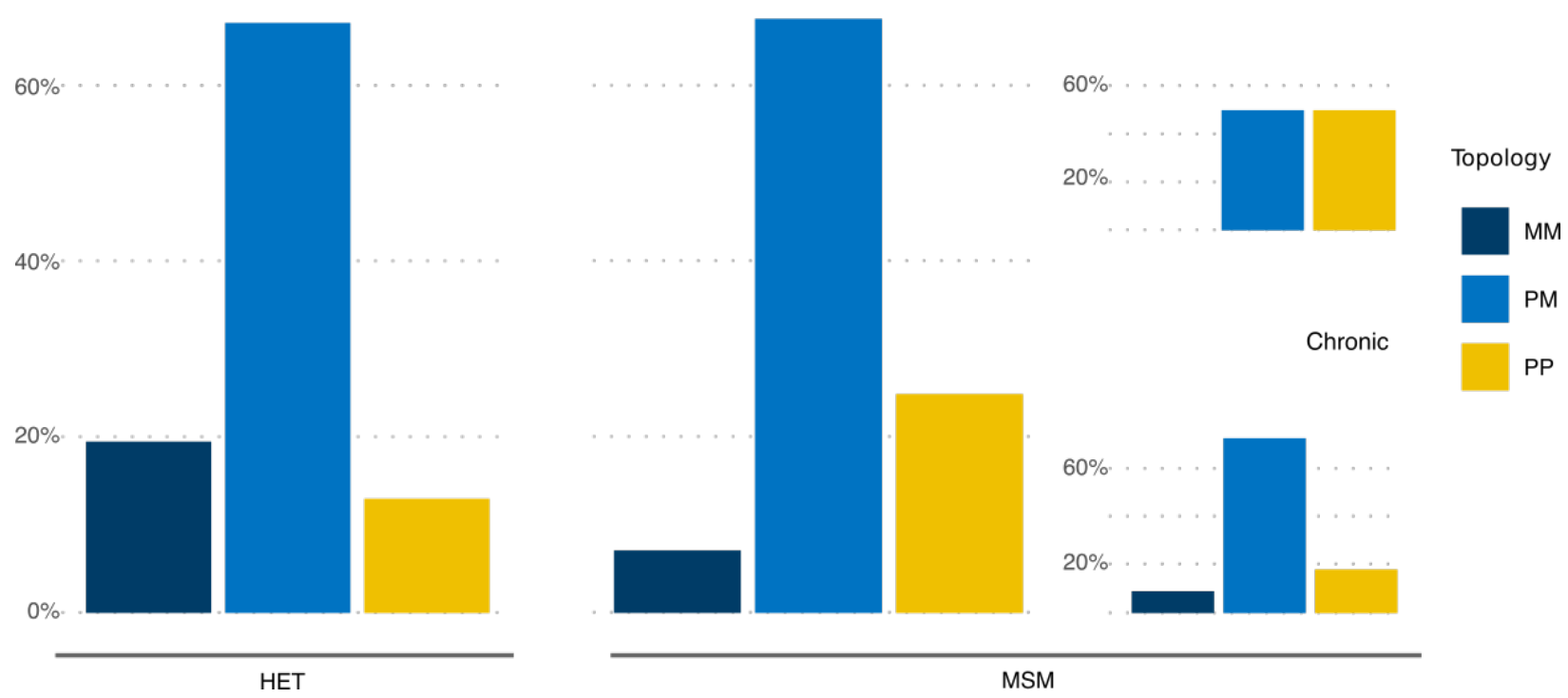

FIGURE S3: Phylogenetic findings of the empirical transmission pairs for whom the posterior trees gave a certainty of over $\mathbf{9 5 \%}$ for the most frequent topology. Fraction of phylogenetic tree topology class (MM Monophyletic-Monophyletic, PM - Paraphyletic-Monophyletic and PP - Paraphyletic-Polyphyletic) where each tree topology class is classified as the most frequent topology class of each posterior distribution per transmission pair. Results are stratified by risk group: 76 heterosexual (HET) pairs and 36 men-who-have-sex-with-men (MSM) pairs) and infection stage of the source partner at transmission (11 acute pairs defined as $<90 \mathrm{~d}$ post infection and 101 chronic pairs defined as $\geq 90 \mathrm{~d}$ post infection).

110 In the main analysis we assumed that all index cases transmit to the source partner after three years of

111 infection. Here we also evaluated the results assuming the transmission risk was skewed towards early

112 infection, with half of all simulations across all transmission pairs assuming index case transmission 
114 qualitatively similar results as our main analysis. The median number of variants transmitted across all

115 pairs is 1 (range: 1-5, Fig. S4A). Across all pairs in both risk groups, the mean probability of observing

116 one founder variant is 0.73 . Stratifying by risk group, we find there is a higher probability that one variant

117 founds HET infections than MSM infections (a geometric mean of 0.79 vs. 0.61 , Fig. S4B). In contrast,

118 when stratifying solely by infection stage of the source partner, we find that transmission during the acute

A

A

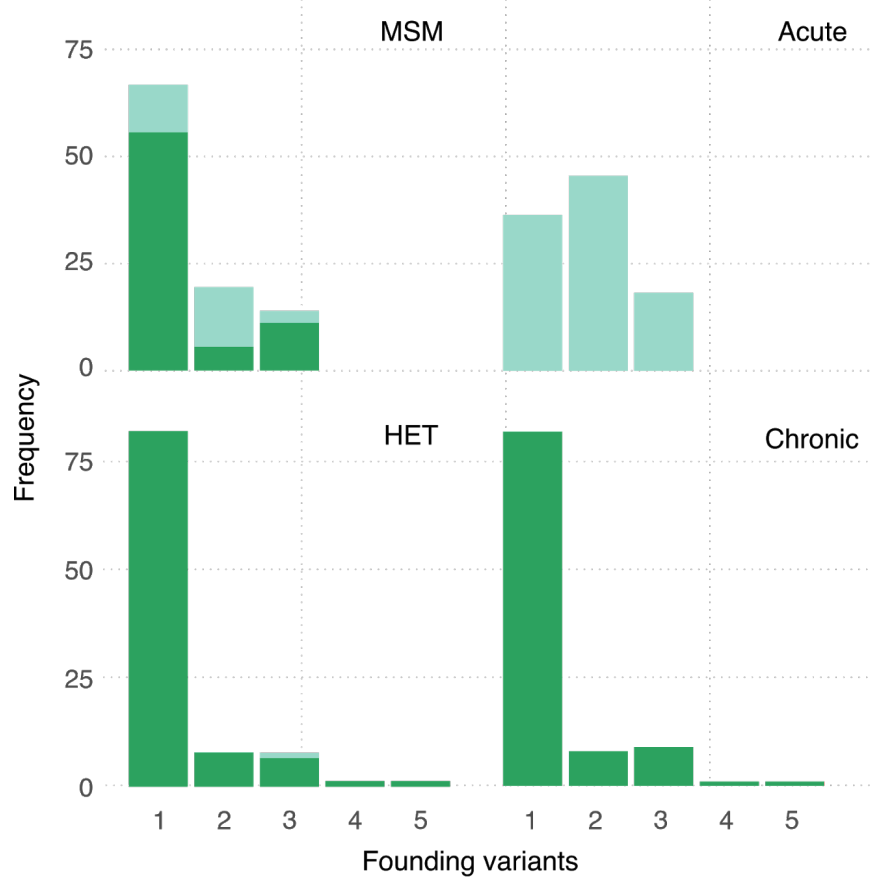

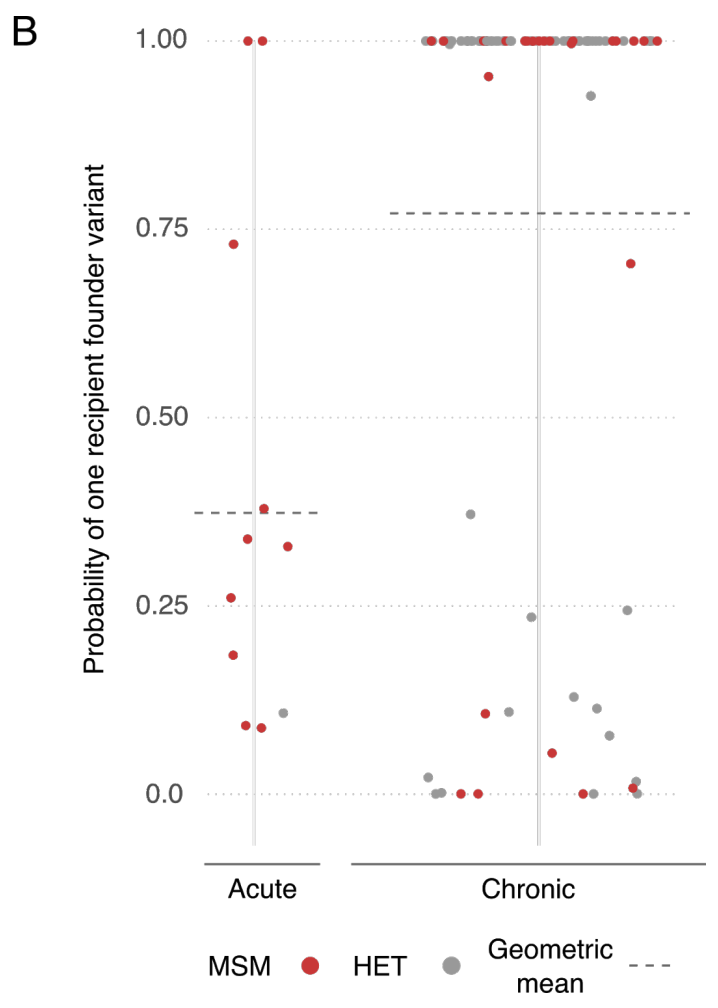

FIGURE S4: Phylogenetic findings from the calibrated simulations with skewed transmission rate towards

acute stage for the index case. A) Frequency of multiplicity of founding variants for transmission pairs by infection stage of source partner at transmission and risk group. The multiplicity is calculated as the modal simulated value. at transmission. 

vs. 0.78) with a higher median number of variants transmitted, when only the most likely multiplicity for each pair is considered ( 2 vs. 1, Fig. S4A). From these results, therefore, there is still approximately twice the chance of multiple variant transmission during acute stage infection across both risk groups (relative risk is 0.48$)$.

\section{Effect of constructing empirical data phylogenetic trees using maximum likelihood inference with}

\section{bootstrapping}

In the main analysis we used Bayesian phylogenetic reconstruction to analyse the empirical sampled genetic data of each empirical transmission pair, using the respective posterior distribution to calculate the frequency of each topology class (MM, PM and PP) r. Here we provide a sensitivity analysis to calculate the tree topology class distribution of the empirical sampled genetic data by maximum likelihood

137 phylogenetic tree construction and bootstrapping. After bootstrapping the empirical data 100 times to 138 calculate the frequency of MM, PM and PP topology classes for each transmission pair, we then proceeded using the same methodology as in the main text. That is, we fit the simulation model

140 (parameterised with the pair-specific data) to the bootstrapped data individually for each transmission pair

141 by comparing the frequencies of tree topology classes. Overall our results remained consistent with our

142 main results, albeit with slightly lower probabilities of observing one founder variant. The median

143 number of variants transmitted across all pairs is 1 (range: 1-11, Fig. S5A). Across all pairs in both risk

144 groups, the mean probability of observing one founder variant is 0.62 . Stratifying by risk group, we find

145 there is a higher probability that one variant founds HET infections than MSM infections (a geometric 146 mean of 0.67 vs. 0.53 , Fig. S5B). Stratifying by infection stage of the source partner, we find there is a

147 much lower probability of one founder variant during the acute stage than during the chronic stage (means 148 of 0.31 vs. 0.66 ) with approximately twice the chance of multiple variant transmission during acute stage 149 infection across both risk groups (relative risk is 0.47 ). 
medRxiv preprint doi: https://doi.org/10.1101/19013524; this version posted December 4, 2019. The copyright holder for this preprint (which was not certified by peer review) is the author/funder, who has granted medRxiv a license to display the preprint in perpetuity. It is made available under a CC-BY 4.0 International license.

A

Acute

Chronic

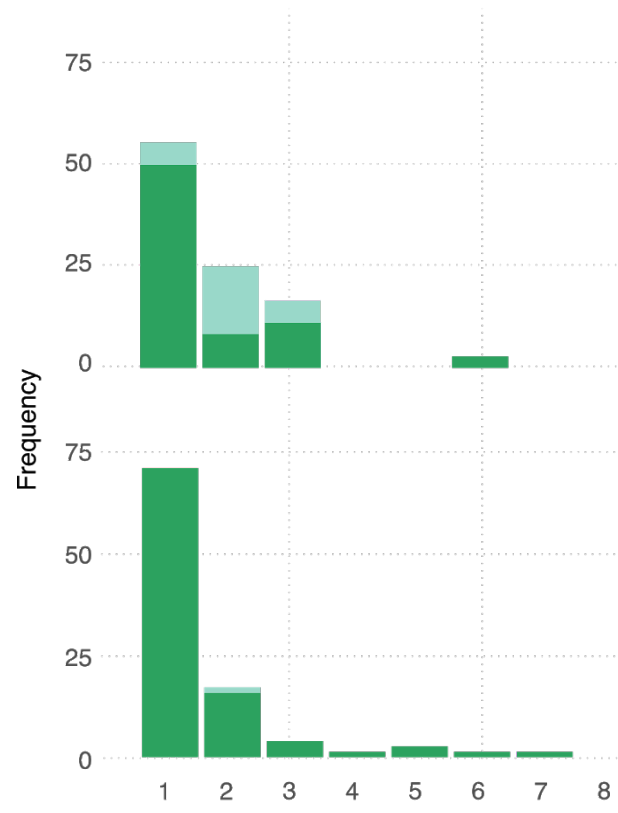

MSM

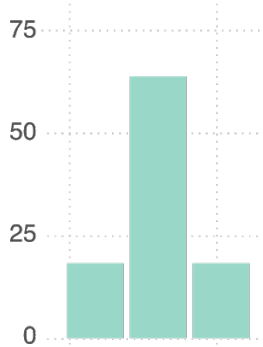

HET

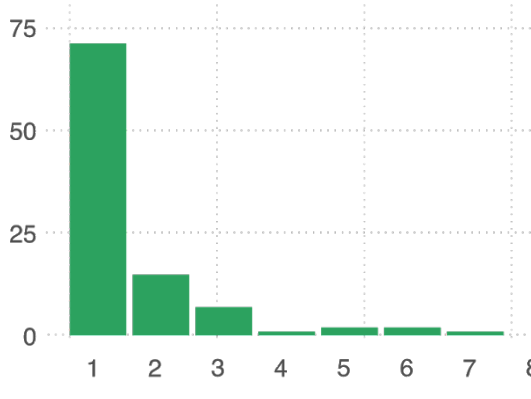

Chronic

\section{B}

Founding variants

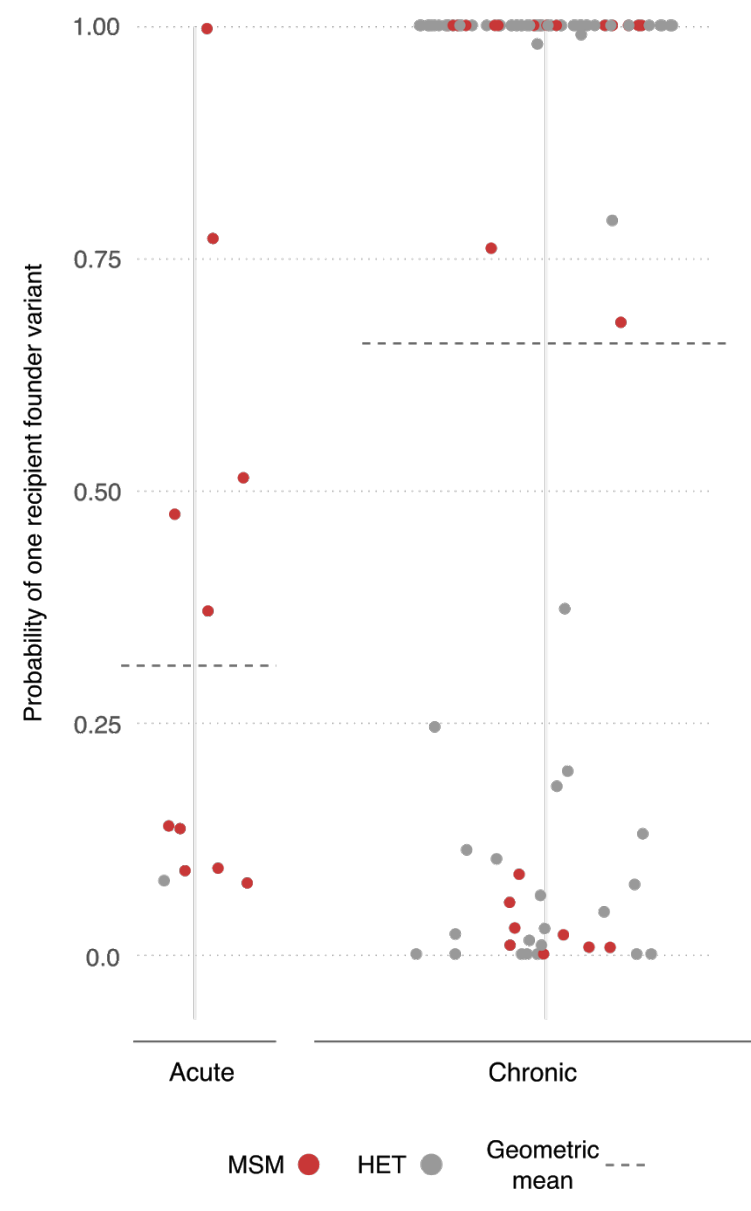


medRxiv preprint doi: https://doi.org/10.1101/19013524; this version posted December 4, 2019. The copyright holder for this preprint (which

was not certified by peer review) is the author/funder, who has granted medRxiv a license to display the preprint in perpetuity.

It is made available under a CC-BY 4.0 International license .

FIGURE S5: Phylogenetic findings from the calibrated simulations with bootstrapped empirical data. A)

152 Frequency of multiplicity of founding variants for transmission pairs by infection stage of source partner at

153 transmission and risk group. The multiplicity is calculated as the modal simulated value. B) Probability of one

154 founder variant in the recipient for each pair stratified by infection stage of the source partner at transmission.

\section{References}

157 Cohen, Myron S., Cynthia L. Gay, Michael P. Busch, and Frederick M. Hecht. 2010. "The Detection of 